\title{
Editorial
}

\section{Martin Franz ${ }^{1}$}

Online publiziert: 18. Dezember 2018

๑) Deutscher Verband für Angewandte Geographie (DVAG) 2018

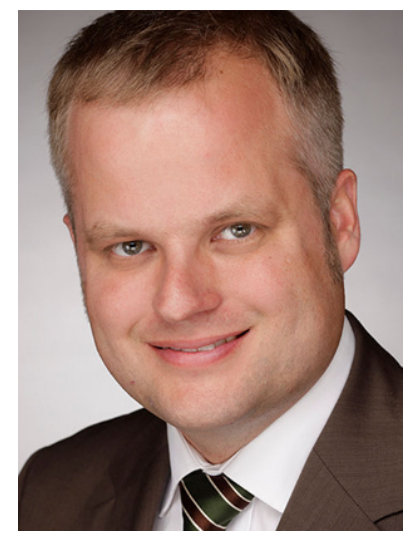

Liebe Leserin, lieber Leser,

Umweltqualität als Standortfaktor, Klimawandelanpassung oder die zunehmende Bedeutung der Green Economy ökologische Fragestellungen stellen für viele Institutionen der Wirtschaftsförderung immer noch neue Herausforderungen dar, deren zunehmende Bedeutung aber offensichtlich ist. Sollen Städte oder Regionen dauerhaft als Wirtschaftsstandorte attraktiv und erfolgreich sein, müssen ökologische Probleme und Chancen mitgedacht werden. In den letzten Jahren zeigten mir Gespräche mit Vertreterinnen und Vertretern von Unternehmen und Wirtschaftsförderungen manches Mal, dass viele Unternehmen eine größere Bereitschaft haben, sich auf neue - ökologischere - Ansätze einzulassen, als es die Akteure der Wirtschaftsförderung und Lokalpolitik erwarten.

Die ökologischere Gestaltung von Gewerbegebieten ist ein gutes Beispiel, an dem sich sehr unterschiedliche Motivationen aufzeigen lassen: Imagefragen spielen sicherlich oft eine Rolle, auch die Erfahrungen mit klimawandelbe-

Martin Franz

Martin.Franz@uos.de

1 Institut für Geographie, Universität Osnabrück, Seminarstr. 19 a/b, 49074 Osnabrück, Deutschland dingten Ereignissen wie Starkregen und Hitze oder der Wunsch, die Attraktivität für Fachkräfte zu steigern. All dies kann dazu führen, dass Unternehmen ihr Interesse an Baumpflanzungen, Entsiegelung oder Fassaden- und Dachbegrünung entdecken. Manche Städte und Regionen haben die Anforderungen an eine nachhaltige Wirtschaftsförderung erkannt, entsprechende Projekte auf den Weg gebracht oder sogar grundlegende Strukturen neu ausgerichtet. Das vorliegende STANDORT-Heft zeigt unterschiedliche Perspektiven auf das Thema auf und stellt Fallbeispiele vor. Jens Clausen und Alexander Schabel betrachten die sogenannte Green Economy in Bezug auf Gründungsförderung, Technologiezentren und Clusterförderung. David Heimann untersucht die Bedeutung von Unternehmensnetzwerken für die nachhaltige Entwicklung von Gewerbegebieten und geht der Frage nach, ob und wie vertikale Netzwerke zwischen Unternehmen und Verwaltung gestärkt werden können. Der Artikel von David Heimann und Jan Stockhorst analysiert am Beispiel eines Konversionsprojektes in der Stadt Rheine, ob die Wirtschaftsförderung als Vermittler verschiedener Interessen und Vernetzer der Akteure zu einer nachhaltigen Stadtentwicklung beiträgt.

Schließlich widmen sich gleich zwei Beiträge der Stadt Augsburg: Während Gesa Pflitsch und Simone Strambach die Transitionspfade verfolgen, die in Augsburg zu einer nachhaltigen Ausrichtung geführt haben, stellt Andreas Thiel in seinem Praxisbericht dar, wie in Augsburg Nachhaltigkeit als roter Faden der Arbeit der Wirtschaftsförderung dient.

Mit besten Grüßen

Martin Franz

P.S.: Aktuell ist erneut der DVAG-Preis für Angewandte Geographie ausgeschrieben. Er wird für hervorragende universitäre Abschlussarbeiten auf dem Gebiet der Angewandten Geographie vergeben. Mehr dazu in diesem Heft in der Rubrik DVAG Intern. 